\title{
ON SOME CLASSES OF SEQUENCES DEFINED BY SEQUENCES OF ORLICZ FUNCTIONS
}

\author{
M. Et, Y. Altin, B. ChOUdhary and B. C. TRIPATHY
}

Abstract. In this paper we introduce some new seminormed sequence spaces using a sequence of Orlicz functions and examine some properties of these sequence spaces. Furthermore we introduce $\Delta_{u_{g}}^{m}$ - statistical convergence and give a relation between $\Delta_{u_{g}}^{m}$ - statistical convergence and strongly $\Delta_{u g}^{m}-$ Cesàro summable sequences with respect to an Orlicz function.

Mathematics subject classification (2000): 40A05, 40C05, 46A45.

Key words and phrases: Orlicz function, sequence spaces, seminorm, statistical convergence.

\section{REFERENCES}

[1] B. Choudhary, S. D. Parashar, A sequence space defined by Orlicz functions, Approx. Theory Appl., 18, (2002), 70-75.

[2] M. ET, R. COLAK, On generalized difference sequence spaces, Soochow J. Math., 21, (1995), $377-386$.

[3] H. FAST, Sur la convergence statistique, Colloq. Math., 2, (1951), 241-244.

[4] J. A. FRIDY, On statistical convergence, Analysis, 5, (1985), 301-313.

[5] P. K. Kamthan, M. Gupta, Sequence Spaces and Series, Marcel Dekker Incorporated, New York, 1981.

[6] H. KIZMAZ, On certain sequence spaces, Canad. Math. Bull., 24, (1981), 169-176.

[7] M. A. KrasnoselkiI, Y. B. Rutitsky, Convex Functions and Orlicz Spaces, Groningen, Netherlands, 1961.

[8] K. LindBerg, On subspaces of Orlicz sequence spaces, Studia Math., 45, (1973), 119-146.

[9] J. Lindenstrauss, L.TZAFRIRI, On Orlicz sequence spaces, Israel J. Math., 10, (1971), 379-390.

[10] I. J. MADDOX, Spaces of strongly summable sequences, Quart. J. Math. Oxford Ser., 18, (1967), 345-355.

[11] N. P. Nung, P. Y. LEE, Orlicz sequence spaces of a nonabsolute type, Comment. Math. Univ. St. Paul., 26, 2 (1977), 209-213.

[12] W. ORLICZ, Linear Functional Analysis (Transl. from the Chinese by Lee Peng Yee, Addendum by W. Congxin ), World Scientific Publishing Company, Singapore, 1992.

[13] T. ŠALAT, On statistically convergent sequences of real numbers, Math. Slovaca, 30, (1980), 139-150.

[14] I. J. ScHOENBERG, The integrality of certain functions and related summability methods, Amer. Math. Monthly, 66, (1959), 361-375.

[15] B. C. TRIPATHY, S. MAhANTA, On a class of sequences related to the $\ell^{p}$ spaces defined by Orlicz. function, Soochow J. Math., 29, 4 ( 2003), 379-391.

[16] J. Y. T. Woo, On modular sequence spaces, Studia Math., 48, (1973), 271-289. 